\title{
Latency-aware secure transmission in MEC-assisted HSR communication systems
}

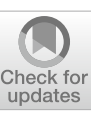

Hao Xu*, Ke Li, Jianfeng Cheng, Bo Jiang and Huai Yu

*Correspondence:

18612113434@163.com

China Academy of Railway

Sciences, Beijing, China

\begin{abstract}
Mobile edge computing can provide short-range cloud computing capability for the mobile users, which is considered to be a promising technology in $5 \mathrm{G}$ communication. The mobile users offload some computing tasks to the edge server through the wireless backhaul link, which can reduce the energy consumption and the time latency. Meanwhile, due to the open characteristics of the wireless channel, the offloading tasks through the backhaul link may face the risk of eavesdropping. Therefore, the secure transmission based on physical layer security for the offloading tasks to the edge server is considered. The optimization problem of minimizing the energy consumption for the vehicular stations (VSs) in mobile edge computing-assisted high-speed railway communication system is studied in this paper. The energy consumption of the mobile users is generated by executing the local computing task and by transmitting the partial offloading task to the edge server. In this paper, a novel joint iterative optimization algorithm is proposed. By jointly optimizing the task scheduling, the task offloading and the transmission power, the energy consumption of all VSs is minimized under the constraint of the time latency. Numerical simulation results verify the effectiveness of the proposed algorithm.
\end{abstract}

Keywords: Mobile edge computing, Backhaul link, Offload, Secure transmission, Energy consumption

\section{Introduction}

\subsection{Challenges and contributions}

With the development of $5 \mathrm{G}$ wireless communication and the Internet of things (IoTs), new technologies such as virtual reality (VR), augmented reality (AR) and unmanned driving appear [1-3]. These technologies require higher data transmission rate and lower time latency. Although new mobile devices are becoming more and more powerful in terms of central processing units (CPUs), they cannot handle applications that require a lot of computing power during a short time. In addition, due to the limited battery energy capacity, high energy consumption is still an important reason for limiting users to deal with high computing applications [4-6]. These factors promote the development of mobile cloud computing (MCC) [7]. MCC transfers the computing load of applications to the cloud servicer and provides complex applications for the mobile users [8, 9]. The high-speed and reliable air interface allows the computing services of mobile devices

(c) The Author(s) 2021. Open Access This article is licensed under a Creative Commons Attribution 4.0 International License, which permits use, sharing, adaptation, distribution and reproduction in any medium or format, as long as you give appropriate credit to the original author(s) and the source, provide a link to the Creative Commons licence, and indicate if changes were made. The images or other third party material in this article are included in the article's Creative Commons licence, unless indicated otherwise in a credit line to the material. If material is not included in the article's Creative Commons licence and your intended use is not permitted by statutory regulation or exceeds the permitted use, you will need to obtain permission directly from the copyright holder. To view a copy of this licence, visit http:// creativecommons.org/licenses/by/4.0/. 
to run in the remote cloud service center [10]. However, MCC has an inherent limitation, that is, the distance from the mobile users to the remote cloud center is relatively far, which will lead to too long latency of the mobile applications. Therefore, MCC is difficult to meet various emerging mobile applications that are critical to latency, and it is difficult to achieve the millisecond delay of 5G computing and communication [11-13].

In order to solve the long latency in MCC, cloud services are moved near the mobile users to provide the cloud computing in the wireless access network, which is the socalled mobile edge computing (MEC) [14, 15]. The concept of MEC is first proposed by European Telecommunications Standards Association (ETSI) in 2014. The initial purpose is to offload the computing tasks on the mobile devices to the base stations [16]. MEC integrates the functions of the base stations and physical servers and offloads tasks to the edge server nodes, which facilitates the mobile users to perform computing-intensive and delay critical tasks on the mobile devices. Therefore, proper task scheduling and offloading can bring more energy savings and lower latency [17].

Due to the open characteristics of the wireless channel, when the mobile user offloads the task to the edge server through the backhaul link and the server feeds back the computing results to the mobile user, the data on the backhaul link in the transmission process are at risk of being eavesdropped. Physical layer security is considered as an effective method to ensure the security of the wireless transmission [18]. From the perspective of information theory, the essence of the physical layer secure transmission is to maximize the security rate of the system. The security rate is defined as the rate of the legitimate users minus the rate of the eavesdropping users [19].

With the popularization of the intelligent terminals and the wide application of IoTs, the energy consumption generated by the information and communication technology is gradually increasing, and the resulting carbon dioxide emissions account for an increasing proportion of global greenhouse gas emissions. Green communication has become an important index for the design and implementation of wireless communication in the future [20]. By offloading some tasks to the edge server, MEC can effectively reduce the energy consumption of the users and improve the life of the users' batteries, which has become a promising technology in the future green communication.

In this paper, we mainly study the task offloading problem of minimizing the energy consumption of the vehicular stations (VSs) in MEC-assisted high-speed railway (HSR) wireless communication system. The energy consumption of each VS consists of two parts. The first part is the energy consumed by VS when executing the local computing tasks, and the second part is the energy consumed by safely transmitting offloaded computing tasks to the relevant edge servers. The energy consumption caused by executing local computing tasks is related to the amount of tasks that VS need to schedule, the offloading ratio, the CPU frequency, the number of CPU cycles, etc. The factors related to the energy consumption generated when transmitting offloading computing tasks include: the amount of tasks that VS needs to schedule, the offloading ratio, the wireless channel state information, the inter channel interference, the transmission power, the system bandwidth, the interference by the eavesdropping user, the energy consumption of the hardware equipments, etc.

For MEC-assisted HSR wireless communication system, we propose an iterative algorithm to minimize the energy consumption of multiple VSs, while ensuring the latency 
constraints and the power constraints of the computing tasks. The main contributions of this paper are as follows:

(1) The MEC-assisted HSR wireless communication system is considered, and the energy consumption minimization of multiple VSs based on task offloading is studied. The optimization variables include the amount of the scheduled task of each VS, the task offloading ratio and the transmission power. The constraints conditions include the local computing time, the task offloading time, the security rate, the transmission power, the task offloading ratio and so on.

(2) Because the optimization variables task scheduling, task offloading ratio and transmission power are coupled with each other, the optimization problem of minimizing energy consumption is difficult to solve. By fixing two of the three variables, we decompose the original problem into three sub-problems, namely task scheduling sub-problem, task offloading ratio sub-problem and power control sub-problem.

(3) For the task scheduling sub-problem, a solution based on interior point method is proposed. For the task offloading ratio sub-problem, we give the closed form expression of the optimal solution. For the non-convex power control sub-problem, by introducing the auxiliary variables and using the successive convex approximation, the non-convex optimization problem is transformed into a convex optimization one, which can be easily solved by CVX. At last, a novel iterative algorithm for minimizing energy consumption is proposed.

The rest of this paper is organized as follows. In Sect. 2, the MEC-assisted HSR communication system model and the latency-aware energy optimization problem are presented. According to the presented system model, Sect. 3 designs an efficient iterative algorithm to minimize the energy consumption problem which is a joint optimization problem of task scheduling, offloading and power control. Then, the simulation results and discussion are given in Sect. 4. Finally, Sect. 5 concludes this paper.

\subsection{Related work}

Although the mobile devices have been very intelligent now, they are still limited by the computing ability and battery capacity. Some applications which need a large amount of computing workload lead to much battery energy consumption. In addition to the limited battery capacity, the CPU processing, memory and storage capacity will also affect the smooth execution of some applications on the mobile devices. The computing-intensive tasks can be offloaded to the adjacent cloud servers or the edge servers, and the servers are all equipped with powerful computing resources.

The computing offloading problem in MEC for a single user was investigated in prior works. The minimization of energy consumption of the mobile users under the constraints of the average latency was considered in [21]. Using the dynamic offline programming method, the two solutions of the deterministic one and the random one were designed to find the optimal wireless scheduling and task offloading strategy. Simulation results showed that the dynamic offline programming algorithm could make the mobile users get the optimal energy efficiency. A fine-granularity task offloading method to minimize the system energy consumption was proposed in [22], and the strict latency 
constraint was also ensured. A practical multi-task application was modeled as a general graph topology. The original task offloading problem was transformed into a mathematical 0-1 programming problem. A binary particle swarm optimization (BPSO) algorithm with a low computational complexity was proposed to solve this problem. Simulation results showed that the proposed algorithm could save more energy than the simple local execution strategy and the traditional rough-granularity task offloading strategy. The base station could transmit energy to the mobile device or receive the computing offloading from the mobile device, and the single mobile device could harvest energy to be local computing or offload data to the base station [23]. Under the constraints of energy harvesting and deadline, the optimization goal of the designed strategy was to maximize the probability of successfully computing the given data. The optimization problem was equivalently transformed into minimizing the energy consumption caused by local computing and maximizing the energy saving caused by offloading, and the closed solution was given. The joint task scheduling and computing offloading for multicomponent applications were introduced in [24], and the component offloading and the scheduling order were studied. The proposed optimization decision strategy changed from the predetermined compiler scheduling order to the radio scheduling order. For some component correlation graph structures, by processing some components in parallel in the mobile device and the cloud, the proposed strategy reduced the execution time of the components. The cooperative task execution between a mobile device and a cloud server in random wireless channel was investigated in [25]. A mobile application consisted of a series of tasks that could be processed on the mobile device or the cloud server. An efficient scheduling strategy was designed to minimize the energy consumption of mobile devices under the minimum latency constraint. For a practical sequentially executed task model, the proposed exhaustive search method obtained the optimal solution, and the proposed adaptive lagrangian relaxation-based aggregated cost algorithm achieved the approximate solution.

The computing offloading and resource allocation for multi-user are further studied in some recent works. Labidi et al. [26] studied minimizing the energy consumption in multi-user small cell network systems through joint radio resource scheduling and computing offloading. An optimal scheduling and offloading method was proposed by using the deterministic method. In this method, only one user was selected for scheduling and computing offloading at each time slot, and the other mobile users were local computing or idle state. Zhang [27] considered the MEC-assisted multi-user multi-task 5G heterogeneous network; an optimization problem to minimize the system energy consumption was formed while satisfying the latency constraint. The system energy consumption was generated by task computation and partial offloading task transmission. In order to reduce the complexity of multi access characteristics, a three-stage computing offloading scheme was designed. This scheme adopted the type classification and priority allocation for the mobile devices; the energy-efficient optimization problem could be solved with polynomial complexity. A computing offloading strategy based on improved particle swarm optimization (PSO) method was proposed for MEC-assisted 5G communication system in [28]. The latency minimization optimization problem was formulated, and the energy consumption was the constraint condition. By introducing the penalty function to balance the time delay and the energy consumption, the proposed computational 
offloading algorithm could reasonably allocate the offloading task to the related edge server. Simulation results showed that under the same energy constraints, compared with all local execution algorithm, MEC random algorithm that randomly assigns the execution task between the local user and the MEC server, and particle swarm optimization algorithm, the proposed algorithm had lower system cost. A multiple users single edge server computing offloading system was considered, and each user had different computing offloading [29]. Under the constraint of computation latency, the weighted sum of energy consumption of mobile users was a convex optimization problem. For the derived offloading priority function, the optimal algorithm has a threshold based structure. According to the user's channel state information and the energy consumption generated by local computation, the algorithm assigned different priorities to the users and performed different computing offloading. In order to reduce the iterative complexity of the algorithm, a suboptimal resource allocation algorithm was also proposed. The minimizing energy consumption through the resource allocation in wireless cloud access network was researched in [30]. Under the constraints of task execution time, transmission power, computing capacity and front-end transmission data rate, the formulated energy consumption minimization problem was a nonconvex optimization one. The nonconvex optimization problem was reconstructed into an equivalent convex optimization one by the weighted least mean square error method. Simulation results showed that the proposed iterative algorithm reduced energy consumption and improved system performance. Multiple mobile users multi-cell multiple-in multiple-out (MIMO) system with a cloud server was considered in [31]; the energy consumption minimization problem with the latency constraint by jointly optimizing the transmitting precoding matrices and the CPU cycles was studied. The formulated optimization problem was non-convex and intractable to solve. A novel iterative algorithm by using successive convex approximation was proposed to achieve a local optimal solution, and the proposed algorithm could run in a distributed manner at multiple wireless access points.

In this paper, the computing offloading strategy of multiple VSs and multiple edge server in MEC-assisted HSR communication system is considered; under the constraints of the safe computing task offloading, transmission latency and transmission power, a novel iterative algorithm to minimize the total energy consumption of multiple VSs is proposed.

\section{Methods}

\subsection{System model}

We consider a MEC-assisted HSR communication system that consists of $K$ single antenna VSs installed on the train roof, the roadside base station (RBS) having a MEC server and one eavesdropper. All RBSs are deployed at regular intervals and the set of VSs is defined as $\mathcal{K}=\{1,2, \ldots, K\}$. All base stations and the eavesdropper are configured with single antenna. Suppose that the running speed of the train is a uniform speed $v$ within a distance. In this region, the running time is equally split into $N$ time slots for the sake of convenience. Thus, we get the equation $L=N T v$ where $T$ is the length of a time slot, $L$ is the running distance and the set of time slots is defined as $\mathcal{N}=\{1,2, \ldots, N\}$. The system model is illustrated in Fig. 1. 


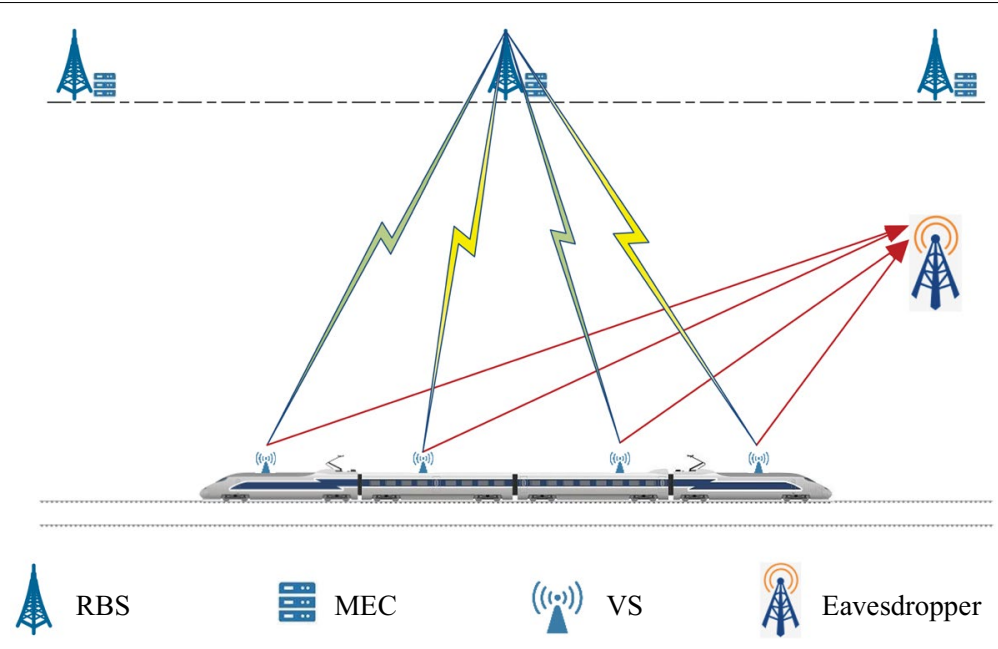

Fig. 1 System model for secure MEC-assisted HSR communication

In time slot $n$, VS $k$ has the tasks $m_{k}[n]$ to be processed. With the partial offloading protocol, the computing tasks are divided into two parts: $\eta_{k}[n] m_{k}[n]$ offloading tasks that are securely offloaded to the RBS and executed by MEC server and $\left(1-\eta_{k}[n]\right) m_{k}[n]$ local computing tasks that are computed locally where $\eta_{k}[n]$ is the offloading ratio. To avoid interference among VSs during tasks offloading, each time block is equally divided into $K$ time slots where each time slot only allows one VS to offload its tasks to the RBS. Thus, for each VS, $\frac{T}{K}$ is the time spent on offloading in each time slot $n \in \mathcal{N}$.

For VS $k$ in time slot $n$, the local computing time can be expressed as

$$
\tau_{k}^{\mathrm{loc}}[n]=\frac{\left(1-\eta_{k}[n]\right) m_{k}[n] C_{k}[n]}{f_{k}[n]}
$$

where $f_{k}[n]$ denotes the CPU frequency of VS $k$ and $C_{k}[n]$ is the number of CPU cycles required for VS $k$ to compute one bit of computing tasks locally [32].

For VS $k$ in time slot $n$, the energy consumption for executing local computing tasks can be represented as

$$
E_{k}^{\mathrm{loc}}[n]=\left(1-\eta_{k}[n]\right) m_{k}[n] C_{k}[n] \varpi_{k} f_{k}^{2}[n],
$$

where $\varpi_{k}$ is the effective capacitance coefficient which depends on the chip architecture at VS $k$ [33].

We consider the inter-channel interference (ICI) caused by Doppler effect to the highmobility train, which is treated as a part of white noise. Thus, the Doppler interference factor $P_{\mathrm{ICI}}$ is expressed as

$$
P_{\mathrm{ICI}}=1-\int_{-1}^{1}(1-|\tau|) J_{0}\left(2 \pi f_{\mathrm{d}} T_{\mathrm{s}} \tau\right) \mathrm{d} \tau
$$

where $J_{0}(\cdot)$ is the zeroth-order Bessel function of the first kind, $f_{\mathrm{d}}$ is the maximum Doppler frequency and $T_{\mathrm{s}}$ is the symbol duration. 
When VS $k$ needs to transmit the tasks to the associated RBS in time slot $n$, the achievable data rate of the uplink transmission from VS $k$ is given by

$$
R_{k}^{\mathrm{VS}}[n]=\log _{2}\left(1+\frac{p_{k}[n] h_{k}[n]}{P_{\mathrm{ICI}} h_{k}[n]+\sigma_{k}^{2}}\right),
$$

where $p_{k}[n]$ denotes the transmission power from VS $k$ to the associated RBS at time slot $n, h_{k}[n]$ represents the uplink channel gain from VS $k$ to the associated RBS at time slot $n$ and $\sigma_{k}^{2}$ is the noise power.

The data rate for the eavesdropper to eavesdrop the signal from the VS $k$ can be given as

$$
R_{k}^{\mathrm{eav}}[n]=\log _{2}\left(1+\frac{p_{k}[n] g_{k}[n]}{P_{\mathrm{ICI}} g_{k}[n]+\sigma_{e}^{2}}\right),
$$

where $g_{k}[n]$ represents the uplink channel gain from VS $k$ to the eavesdropper at time slot $n$ and $\sigma_{e}^{2}$ is the noise power.

The secrecy rate is given by [34]

$$
R_{k}[n]=\left[R_{k}^{\mathrm{VS}}[n]-R_{k}^{\mathrm{eav}}[n]\right]^{+},
$$

where $[x]^{+} \triangleq \max (x, 0)$.

For VS $k$ in time slot $n$, the offloading time can be expressed as

$$
\tau_{k}^{\mathrm{off}}[n]=\frac{\eta_{k}[n] m_{k}[n]}{B R_{k}[n]},
$$

where $B$ is network bandwidth.

For VS $k$ in time slot $n$, the energy consumption for transmitting the computing tasks to the associated RBS can be represented as

$$
E_{k}^{\mathrm{off}}[n]=p_{k}[n] \tau_{k}^{\mathrm{off}}[n]+\frac{T}{K} P_{c},
$$

where $P_{c}$ represents the hardware power dissipated in all other hardware components of the communication system.

It is assumed that the computing capability of MEC server is infinite and the time spending on MEC and the downlink transmission is disregarded. The total energy consumption for VS $k$ in time slot $n$ can be given by

$$
\begin{aligned}
E_{k}[n]= & E_{k}^{\mathrm{loc}}[n]+E_{k}^{\mathrm{off}}[n] \\
= & \left(1-\eta_{k}[n]\right) m_{k}[n] C_{k}[n] \varpi_{k} f_{k}^{2}[n]+\frac{T}{K} P_{c} \\
& +\frac{p_{k}[n] \eta_{k}[n] m_{k}[n]}{B\left[\log _{2}\left(1+\frac{p_{k}[n] h_{k}[n]}{P_{\mathrm{ICI} h_{k}[n]+\sigma_{k}^{2}}}\right)-\log _{2}\left(1+\frac{p_{k}[n] g_{k}[n]}{P_{\mathrm{ICI} g_{k}[n]+\sigma_{e}^{2}}}\right)\right]^{+}} .
\end{aligned}
$$




\subsection{Problem formulation}

In this paper, we jointly optimize the task scheduling $\mathbf{m} \triangleq\left\{m_{k}[n], \forall k, n\right\}$, the offloading ratio $\eta \triangleq\left\{\eta_{k}[n], \forall k, n\right\}$ and the power control vector $\mathbf{p} \triangleq\left\{p_{k}[n], \forall k, n\right\}$, so that the sum of all VSs' energy consumption during the time $T$ is minimized. The problem can be formulated as

$$
\begin{aligned}
& P 1: \min _{\mathbf{m}, \eta, \mathbf{p}} \sum_{n=1}^{N} \sum_{k=1}^{K} E_{k}[n] \\
& \text { s.t. } R_{k}[n] \geq R_{k}^{\min }, \quad \forall k \in \mathcal{K}, \forall n \in \mathcal{N}, \\
& \sum_{n \in \mathcal{N}} m_{k}[n] \geq M_{k}, \quad \forall k \in \mathcal{K}, \\
& \tau_{k}^{\text {off }}[n] \leq \frac{T}{K}, \quad \forall k \in \mathcal{K}, \forall n \in \mathcal{N}, \\
& \tau_{k}^{\text {loc }}[n] \leq T, \quad \forall k \in \mathcal{K}, \forall n \in \mathcal{N}, \\
& m_{k}[n] \geq 0, \quad \forall k \in \mathcal{K}, \forall n \in \mathcal{N}, \\
& 0 \leq p_{k}[n] \leq p_{k}^{\max }, \quad \forall k \in \mathcal{K}, \forall n \in \mathcal{N}, \\
& \eta_{k}[n] \in[0,1], \quad \forall k \in \mathcal{K}, \forall n \in \mathcal{N},
\end{aligned}
$$

where $R_{k}^{\min }$ is the minimum quality of service (QoS) requirement for VS $k, M_{k}$ is the task of VS $k$ that needs to be processed in the time $T$, and $p_{k}^{\max }$ represents the maximum transmit power of VS $k$. Constraint (10b) guarantees the QoS of VS $k$. Constraint (10c) is to ensure that the total task from VS $k$ is processed within the time $T$. Constraint (10d) and constraint (10e) indicate that the time spent in local computing and task offloading cannot be more than the maximum tolerance time, respectively.

The joint task scheduling, offloading ratio and power control problem in $P 1$ have some challenges to solve, since three key variables are coupled in $P 1$. To solve $P 1$, we decompose $P 1$ into three kinds of subproblems by fixing two of three classes of variables alternately.

\section{Iteration-based algorithm for MEC-assisted HSR communication systems}

\subsection{Task scheduling subproblem}

When the power control vector $\mathbf{p}$ and the offloading ratio $\eta$ are fixed, the optimization problem $P 1$ can transformed into $K$ task scheduling subproblems, where the subproblem for VS $k$ is given as 


$$
\begin{aligned}
& P 2: \min _{m_{k}[n]} \sum_{n=1}^{N} E_{k}[n] \\
& \text { s.t. } \sum_{n \in \mathcal{N}} m_{k}[n] \geq M_{k}, \\
& \tau_{k}^{\text {off }}[n] \leq \frac{T}{K}, \quad \forall n \in \mathcal{N}, \\
& \tau_{k}^{\text {loc }}[n] \leq T, \quad \forall n \in \mathcal{N}, \\
& m_{k}[n] \geq 0, \quad \forall n \in \mathcal{N},
\end{aligned}
$$

For convenience, the optimization objective in $P 2$ is simplified as

$$
\begin{aligned}
E_{k}[n] & =\left[\left(1-\eta_{k}[n]\right) C_{k}[n] \varpi_{k} f_{k}^{2}[n]+\frac{p_{k}[n] \eta_{k}[n]}{B R_{k}[n]}\right] m_{k}[n]+\frac{T}{K} P_{c} \\
& =\Delta_{k}[n] m_{k}[n]+\frac{T}{K} P_{c}
\end{aligned}
$$

where $\Delta_{k}[n]=\left(1-\eta_{k}[n]\right) C_{k}[n] \varpi_{k} f_{k}^{2}[n]+\frac{p_{k}[n] \eta_{k}[n]}{B R_{k}[n]}$.

The constraint (11c) and constraint (11d) can be rewritten as

$$
\left\{\begin{array}{l}
m_{k}[n] \leq \frac{T f_{k}[n]}{\left(1-\eta_{k}[n]\right) C_{k}[n]} \\
m_{k}[n] \leq \frac{T B R_{k}[n]}{K \eta_{k}[n]}
\end{array}\right.
$$

Therefore, the following inequality is obtained.

$$
0 \leq m_{k}[n] \leq \min \left\{\frac{T f_{k}[n]}{\left(1-\eta_{k}[n]\right) C_{k}[n]}, \frac{T B R_{k}[n]}{K \eta_{k}[n]}\right\}
$$

Since $P 2$ is a linear programming problem, we solve it by using interior point method. By applying the log-barrier method, $P 2$ is transformed into an unconstrained optimization problem where the optimization objective is given by

$$
\begin{aligned}
\Phi\left(m_{k}[n]\right)= & t \sum_{n=1}^{N}\left(\Delta_{k}[n] m_{k}[n]+\frac{T}{K} P_{c}\right) \\
& -\ln \left(-M_{k}+\sum_{n \in \mathcal{N}} m_{k}[n]\right) \\
& -\sum_{n=1}^{N} \ln \left(-m_{k}[n]+\Lambda_{k}[n]\right),
\end{aligned}
$$

where $\Lambda_{k}[n]=\min \left\{\frac{T f_{k}[n]}{\left(1-\eta_{k}[n]\right) C_{k}[n]}, \frac{T B R_{k}[n]}{K \eta_{k}[n]}\right\}$.

By deriving the first-order derivative of $\Phi\left(m_{k}[n]\right)$ with respect to $m_{k}[n]$, the following equation can be obtained as 


$$
\frac{\partial \Phi}{\partial m_{k}[n]}=t \Delta_{k}[n]-\frac{1}{\sum_{n \in \mathcal{N}} m_{k}[n]-M_{k}}+\frac{1}{\Lambda_{k}[n]-m_{k}[n]} .
$$

According to the above derivation, we have

$$
\nabla \Phi\left(m_{k}[n]\right)=\left(t \Delta_{k}[n]-\frac{1}{\sum_{n \in \mathcal{N}} m_{k}[n]-M_{k}}+\frac{1}{\Lambda_{k}[n]-m_{k}[n]}\right)_{n=1}^{N} .
$$

Moreover, the second-order derivative of $\Phi\left(m_{k}[n]\right)$ with respect to $m_{k}[n]$ is given by

$$
\left\{\begin{array}{l}
\frac{\partial^{2} \Phi}{\partial^{2} m_{k}[n]}=\frac{1}{\left(\sum_{n \in \mathcal{N}} m_{k}[n]-M_{k}\right)^{2}}+\frac{1}{\left(\Lambda_{k}[n]-m_{k}[n]\right)^{2}} \\
\frac{\partial^{2} \Phi}{\partial m_{k}[n] \partial m_{k}[l]}=\frac{1}{\left(\sum_{n \in \mathcal{N}} m_{k}[n]-M_{k}\right)^{2}}
\end{array}\right.
$$

The Hessian matrix of $\Phi\left(m_{k}[n]\right)$ is represented as

$$
\begin{aligned}
\mathbf{H}\left(m_{k}[n]\right)= & \frac{1}{\left(\sum_{n \in \mathcal{N}} m_{k}[n]-M_{k}\right)^{2}} \mathbf{I} \\
& +\operatorname{diag}\left(\frac{1}{\left(\Lambda_{k}[1]-m_{k}[1]\right)^{2}}, \cdots, \frac{1}{\left(\Lambda_{k}[n]-m_{k}[n]\right)^{2}}\right) .
\end{aligned}
$$

By applying interior point method to solve $P 2$, the update strategy of task scheduling $\mathbf{m}_{k}$ can be expressed as

$$
\mathbf{m}_{k}^{t}=\mathbf{m}_{k}^{t-1}-\mathbf{H}^{-1}\left(\mathbf{m}_{k}^{t-1}\right) \nabla \Phi\left(\mathbf{m}_{k}^{t-1}\right)
$$

The detailed solving process is described in Algorithm 1.

\subsection{Offloading ratio subproblem}

When the power control vector $\mathbf{p}$ and the task scheduling $\mathbf{m}$ are fixed, $P 1$ can be split to $N$ subproblems, which is equivalently formulated as

$$
\begin{aligned}
& P 3: \min _{\eta_{k}[n]} \sum_{k=1}^{K} E_{k}[n] \\
& \text { s.t. } \tau_{k}^{\text {off }}[n] \leq \frac{T}{K}, \quad \forall k \in \mathcal{K}, \\
& \tau_{k}^{\text {loc }}[n] \leq T, \quad \forall k \in \mathcal{K}, \\
& \eta_{k}[n] \in[0,1], \quad \forall k \in \mathcal{K} .
\end{aligned}
$$

For convenience, the optimization objective in $P 3$ is rewritten as

$$
E_{k}[n]=\left(\frac{p_{k}[n] m_{k}[n]}{B R_{k}[n]}-m_{k}[n] C_{k}[n] \varpi_{k} f_{k}^{2}[n]\right) \eta_{k}[n]+m_{k}[n] C_{k}[n] \varpi_{k} f_{k}^{2}[n]+\frac{T}{K} P_{c} .
$$

The constraint (21b) and constraint (21c) can be rewritten as 


$$
\left\{\begin{array}{l}
1-\frac{T f_{k}[n]}{m_{k}[n] C_{k}[n]} \leq \eta_{k}[n] \\
\eta_{k}[n] \leq \frac{T B R_{k}[n]}{K m_{k}[n]}
\end{array}\right.
$$

It is trivial to verify that the optimal solution of the offloading ratio is given as

$$
\eta_{k}^{\text {opt }}[n]= \begin{cases}\max \left(1-\frac{T f_{k}[n]}{m_{k}[n] C_{k}[n]}, 0\right), & \text { if } p_{k}[n] \geq B R_{k}[n] C_{k}[n] \varpi_{k} f_{k}^{2}[n], \\ \min \left(\frac{T B R_{k}[n]}{K m_{k}[n]}, 1\right), & \text { otherwise, }\end{cases}
$$

for $\forall k \in \mathcal{K}$.

\subsection{Power control subproblem}

When $\mathbf{m}$ and $\eta$ are given, $P 1$ is split to $N K$ subproblems, which is equivalently formulated as

$$
\begin{aligned}
& P 4: \min _{p_{k}[n]} E_{k}[n] \\
& \text { s.t. } R_{k}[n] \geq R_{k}^{\min }, \\
& \tau_{k}^{\text {off }}[n] \leq \frac{T}{K}, \\
& 0 \leq p_{k}[n] \leq p_{k}^{\max } .
\end{aligned}
$$

Constraint (25c) can be rewritten as

$$
\frac{K \eta_{k}[n] m_{k}[n]}{T B} \leq R_{k}[n] .
$$

Since the objective function in $P 4$ is non-convex, $P 4$ is transformed as $P 4.1$ by introducing the auxiliary variables $\chi_{k}[n], k \in \mathcal{K}, n \in \mathcal{N}$.

$$
\begin{aligned}
& P 4.1: \min _{p_{k}[n], \chi_{k}[n]} \frac{\eta_{k}[n] m_{k}[n]}{B} \chi_{k}[n] \\
& \text { s.t. } R_{k}[n] \geq \max \left\{R_{k}^{\min }, \frac{K \eta_{k}[n] m_{k}[n]}{T B}\right\}, \\
& R_{k}[n] \geq \frac{p_{k}[n]}{\chi_{k}[n]} \geq 0, \\
& 0 \leq p_{k}[n] \leq p_{k}^{\max } .
\end{aligned}
$$

In P4.1, constraints (27b) and (27c) are still non-convex due to the non-convexity of the logarithm terms in $R_{k}[n]$ and the coupling of two variables.

For the convenience of calculation, constraint (27c) is rewritten as 


$$
\begin{aligned}
& \log _{2}\left(1+\frac{p_{k}[n] h_{k}[n]}{P_{\mathrm{ICI}} h_{k}[n]+\sigma_{k}^{2}}\right)-\log _{2}\left(1+\frac{p_{k}[n] g_{k}[n]}{P_{\mathrm{ICI}} g_{k}[n]+\sigma_{e}^{2}}\right) \geq \frac{p_{k}[n]}{\chi_{k}[n]} \\
& \Rightarrow \chi_{k}[n]\left(\log _{2}\left(1+\frac{p_{k}[n] h_{k}[n]}{P_{\mathrm{ICI}} h_{k}[n]+\sigma_{k}^{2}}\right)-\log _{2}\left(1+\frac{p_{k}[n] g_{k}[n]}{P_{\mathrm{ICI}} g_{k}[n]+\sigma_{e}^{2}}\right)\right) \geq p_{k}[n] \\
& \Rightarrow \chi_{k}[n]\left(\log _{2}\left(p_{k}[n] h_{k}[n]+P_{\mathrm{ICI}} h_{k}[n]+\sigma_{k}^{2}\right)-\log \left(P_{\mathrm{ICI}} h_{k}[n]+\sigma_{k}^{2}\right)\right. \\
& \left.-\log _{2}\left(p_{k}[n] g_{k}[n]+P_{\mathrm{ICI}} g_{k}[n]+\sigma_{e}^{2}\right)+\log _{2}\left(P_{\mathrm{ICI}} g_{k}[n]+\sigma_{e}^{2}\right)\right) \geq p_{k}[n] \\
& \Rightarrow \chi_{k}[n]\left(\log _{2}\left(p_{k}[n] h_{k}[n]+P_{\mathrm{ICI}} h_{k}[n]+\sigma_{k}^{2}\right)-\log _{2}\left(p_{k}[n] g_{k}[n]+P_{\mathrm{ICI}} g_{k}[n]+\sigma_{e}^{2}\right)\right) \\
& \geq p_{k}[n]+\chi_{k}[n]\left(\log \left(P_{\mathrm{ICI}} h_{k}[n]+\sigma_{k}^{2}\right)-\log _{2}\left(P_{\mathrm{ICI}} g_{k}[n]+\sigma_{e}^{2}\right)\right) \text {. }
\end{aligned}
$$

Constraint (28) is relaxed as the following two constraints by introducing the auxiliary variables $\varphi_{k}[n], k \in \mathcal{K}, n \in \mathcal{N}$.

$$
\begin{aligned}
f_{1}\left(p_{k}[n]\right)-f_{2}\left(p_{k}[n]\right) \triangleq & \log _{2}\left(p_{k}[n] h_{k}[n]+P_{\mathrm{ICI}} h_{k}[n]+\sigma_{k}^{2}\right) \\
& -\log _{2}\left(p_{k}[n] g_{k}[n]+P_{\mathrm{ICI}} g_{k}[n]+\sigma_{e}^{2}\right) \geq \varphi_{k}[n],
\end{aligned}
$$

and

$$
\begin{aligned}
\chi_{k}[n] \varphi_{k}[n] \geq & p_{k}[n]+\chi_{k}[n]\left(\log \left(P_{\mathrm{ICI}} h_{k}[n]+\sigma_{k}^{2}\right)\right. \\
& \left.-\log _{2}\left(P_{\mathrm{ICI}} g_{k}[n]+\sigma_{e}^{2}\right)\right) .
\end{aligned}
$$

Since $f_{1}$ and $f_{2}$ are concave functions, $f_{1}\left(p_{k}[n]\right)-f_{2}\left(p_{k}[n]\right)$ is a form of subtraction of two concave functions. We adopt the successive convex approximation (SCA) technique to re-express $(29)$ in the $(t+1)$-th iteration, which is given by

$$
\begin{aligned}
\log _{2}\left(p_{k}[n] h_{k}[n]+P_{\mathrm{ICI}} h_{k}[n]+\sigma_{k}^{2}\right)-\log _{2}\left(p_{k}[n] g_{k}[n]+P_{\mathrm{ICI}} g_{k}[n]+\sigma_{e}^{2}\right) \geq \varphi_{k}[n] \\
-\Rightarrow \log _{2}\left(p_{k}[n] h_{k}[n]+P_{\mathrm{ICI}} h_{k}[n]+\sigma_{k}^{2}\right) \\
\quad\left(\log _{2}\left(p_{k}^{t}[n] g_{k}[n]+P_{\mathrm{ICI}} g_{k}[n]+\sigma_{e}^{2}\right)+\frac{g_{k}[n]}{p_{k}^{t}[n] \ln 2}\left(p_{k}[n]-p_{k}^{t}[n]\right)\right) \geq \varphi_{k}[n] \\
\Rightarrow \log _{2}\left(p_{k}[n] h_{k}[n]+P_{\mathrm{ICI}} h_{k}[n]+\sigma_{k}^{2}\right)-\frac{g_{k}[n]}{p_{k}^{t}[n] \ln 2} p_{k}[n]-\varphi_{k}[n] \\
\geq \log _{2}\left(p_{k}^{t}[n] g_{k}[n]+P_{\mathrm{ICI}} g_{k}[n]+\sigma_{e}^{2}\right)-\frac{g_{k}[n]}{p_{k}^{t}[n] \ln 2} p_{k}^{t}[n] .
\end{aligned}
$$

The first item in inequality (30) is coupled with respect to the optimization variables. Its first-order Taylor expansion around a feasible point $\left(\chi_{k}^{t}[n], \varphi_{k}^{t}[n]\right)$ at the $(t+1)$-th iteration is given as 


$$
\begin{aligned}
\chi_{k}[n] \varphi_{k}[n]= & \frac{\left(\chi_{k}[n]+\varphi_{k}[n]\right)^{2}-\left(\chi_{k}[n]-\varphi_{k}[n]\right)^{2}}{4} \\
\geq & \frac{\left(\chi_{k}^{t}[n]+\varphi_{k}^{t}[n]\right)\left(\chi_{k}[n]+\varphi_{k}[n]\right)}{2} \\
& -\frac{\left(\chi_{k}^{t}[n]+\varphi_{k}^{t}[n]\right)^{2}}{4}-\frac{\left(\chi_{k}[n]-\varphi_{k}[n]\right)^{2}}{4} .
\end{aligned}
$$

Thus, constraint (30) is derived as a further tight constraint (33) at the $(t+1)$-th iteration.

$$
\begin{aligned}
& \frac{\left(\chi_{k}^{t}[n]+\varphi_{k}^{t}[n]\right) \varphi_{k}[n]}{2}-\frac{\left(\chi_{k}[n]-\varphi_{k}[n]\right)^{2}}{4} \geq p_{k}[n]+\frac{\left(\chi_{k}^{t}[n]+\varphi_{k}^{t}[n]\right)^{2}}{4} \\
& \quad+\chi_{k}[n]\left(\log \left(P_{\mathrm{ICI}} h_{k}[n]+\sigma_{k}^{2}\right)-\log _{2}\left(P_{\mathrm{ICI}} g_{k}[n]+\sigma_{e}^{2}\right)-\frac{\left(\chi_{k}^{t}[n]+\varphi_{k}^{t}[n]\right)}{2}\right) .
\end{aligned}
$$

To convexify (27b), the first-order Taylor expansion of the first two items in (27b) around a feasible point $p_{k}^{t}[n]$ at the $(t+1)$-th iteration is given and (27b) is derived as a further tight constraint (34) at the $(t+1)$-th iteration.

$$
\begin{aligned}
\log _{2} & \left(1+\frac{p_{k}[n] h_{k}[n]}{P_{\mathrm{ICI}} h_{k}[n]+\sigma_{k}^{2}}\right)-\log _{2}\left(1+\frac{p_{k}[n] g_{k}[n]}{P_{\mathrm{ICI}} g_{k}[n]+\sigma_{e}^{2}}\right) \\
\geq & \max \left\{R_{k}^{\min }, \frac{K \eta_{k}[n] m_{k}[n]}{T B}\right\} \\
\Rightarrow & \log _{2}\left(p_{k}[n] h_{k}[n]+P_{\mathrm{ICI}} h_{k}[n]+\sigma_{k}^{2}\right)-\log _{2}\left(p_{k}[n] g_{k}[n]+P_{\mathrm{ICI}} g_{k}[n]+\sigma_{e}^{2}\right) \\
\geq & \max \left\{R_{k}^{\min }, \frac{K \eta_{k}[n] m_{k}[n]}{T B}\right\}+\log \left(P_{\mathrm{ICI}} h_{k}[n]+\sigma_{k}^{2}\right)-\log _{2}\left(P_{\left.\mathrm{ICI} g_{k}[n]+\sigma_{e}^{2}\right)}\right. \\
\Rightarrow & \log _{2}\left(p_{k}[n] h_{k}[n]+P_{\mathrm{ICI}} h_{k}[n]+\sigma_{k}^{2}\right)-\log _{2}\left(p_{k}^{t}[n] g_{k}[n]+P_{\mathrm{ICI}} g_{k}[n]+\sigma_{e}^{2}\right) \\
& -\frac{g_{k}[n]}{p_{k}^{t}[n] \ln 2}\left(p_{k}[n]-p_{k}^{t}[n]\right) \\
\geq & \max \left\{R_{k}^{\min }, \frac{K \eta_{k}[n] m_{k}[n]}{T B}\right\}+\log \left(P_{\mathrm{ICI}} h_{k}[n]+\sigma_{k}^{2}\right)-\log _{2}\left(P_{\left.\mathrm{ICI} g_{k}[n]+\sigma_{e}^{2}\right) .}\right.
\end{aligned}
$$

According to the above analysis, problem $P 4.1$ is reformulated as P4.2, i.e.,

$$
\text { P4.2: } \min _{p_{k}[n], \chi_{k}[n], \varphi_{k}[n]} \frac{\eta_{k}[n] m_{k}[n]}{B} \chi_{k}[n]
$$

s.t. (31), (33), (34),

$$
0 \leq p_{k}[n] \leq p_{k}^{\max },
$$

which is a convex optimization problem and can be solved by CVX effectively. 


\subsection{Algorithm description}

In Algorithm 1, the task scheduling $\mathbf{m}$ with the given $\boldsymbol{\eta}$ and $\mathbf{p}$ is optimized, and in Algorithm 2, the joint offloading ratio and power control $(\boldsymbol{\eta}, \mathbf{p})$ with the given $\mathbf{m}$ are solved. The joint iterative optimization procedure is presented in Algorithm 3.
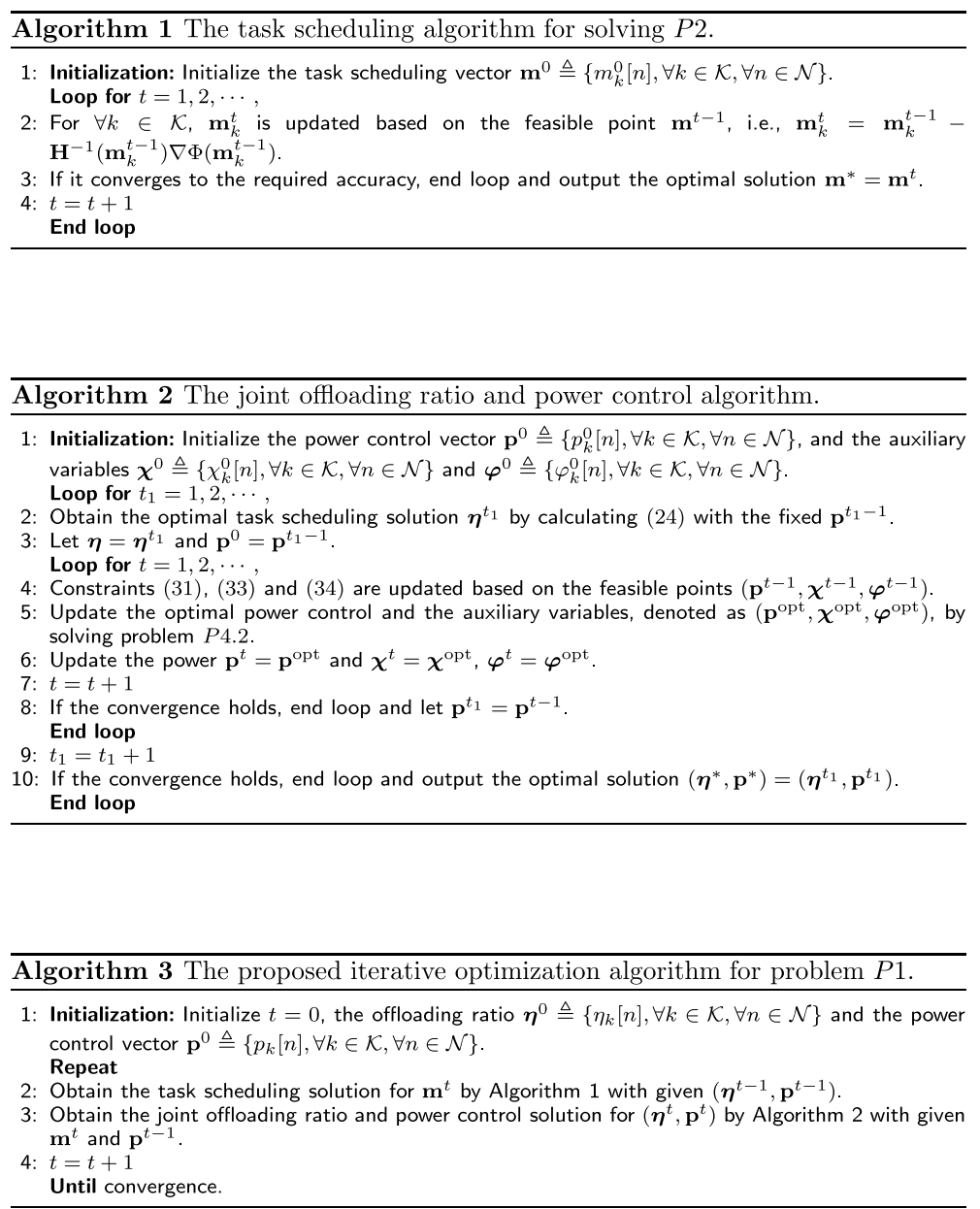

In order to verify the convergence of Algorithm 3 , we define $\phi_{1}(\mathbf{m}, \boldsymbol{\eta}, \mathbf{p})$ as the objective value of $P 1$, and define $\phi_{2}(\mathbf{m}, \boldsymbol{\eta}, \mathbf{p}), \phi_{3}(\mathbf{m}, \boldsymbol{\eta}, \mathbf{p}), \phi_{4}(\mathbf{m}, \boldsymbol{\eta}, \mathbf{p})$ and $\phi_{4.2}(\mathbf{m}, \boldsymbol{\eta}, \mathbf{p})$ as the sum of the objective values for all $k \in \mathcal{K}$ in $P 2$, all $n \in \mathcal{N}$ in $P 3$, and all $k \in \mathcal{K}, n \in \mathcal{N}$ in $P 4$ and $P 4.2$, respectively.

In step 5 of Algorithm 2, we can obtain the solution $\mathbf{p}^{t}$ to $P 4.2$ with the feasible points $\mathbf{p}^{t-1}, \chi^{t-1}$ and $\varphi^{t-1}$. As the iterative number increasing in step 6 and step 7 , the feasible points are updated and the feasible region is enlarged with the SCA method. When $\mathbf{p}^{t}=\mathbf{p}^{t-1}$, we have $\phi_{4.2}\left(\mathbf{p}^{t-1}, \mathbf{m}^{t-1}, \boldsymbol{\eta}^{t-1}\right) \geq \phi_{4.2}\left(\mathbf{p}^{t}, \mathbf{m}^{t-1}, \boldsymbol{\eta}^{t-1}\right)$, where $\mathbf{p}^{t}$ is the globally optimal solution for $P 4.2$ with fixed $\mathbf{m}^{t-1}$ and $\eta^{t-1}$. Since $P 4.2$ is equivalent to $P 4$, the inequality $\phi_{4}\left(\mathbf{p}^{t-1}, \mathbf{m}^{t-1}, \boldsymbol{\eta}^{t-1}\right) \geq \phi_{4}\left(\mathbf{p}^{t}, \mathbf{m}^{t-1}, \eta^{t-1}\right)$ holds. In Algorithm 2, the obtained solutions between inner loop and outer loop have the following relationship: 
$\phi_{3}\left(\mathbf{p}^{t-1}, \mathbf{m}^{t-1}, \eta^{t-1}\right) \geq \phi_{3}\left(\mathbf{p}^{t-1}, \mathbf{m}^{t-1}, \eta^{t}\right)=\phi_{4}\left(\mathbf{p}^{t-1}, \mathbf{m}^{t-1}, \eta^{t}\right) \geq \phi_{4}\left(\mathbf{p}^{t}, \mathbf{m}^{t-1}, \boldsymbol{\eta}^{t}\right)=$ $\phi_{3}\left(\mathbf{p}^{t}, \mathbf{m}^{t-1}, \eta^{t}\right)$

That is, $\phi_{3}\left(\mathbf{p}^{t-1}, \mathbf{m}^{t-1}, \boldsymbol{\eta}^{t-1}\right) \geq \phi_{3}\left(\mathbf{p}^{t}, \mathbf{m}^{t-1}, \boldsymbol{\eta}^{t}\right)$.

In Algorithm 3, the obtained solutions in step 2 and step 3 have the following relationship: $\quad \phi_{1}\left(\mathbf{p}^{t-1}, \mathbf{m}^{t-1}, \boldsymbol{\eta}^{t-1}\right)=\phi_{2}\left(\mathbf{p}^{t-1}, \mathbf{m}^{t-1}, \boldsymbol{\eta}^{t-1}\right) \geq \phi_{2}\left(\mathbf{p}^{t-1}, \mathbf{m}^{t}, \boldsymbol{\eta}^{t-1}\right)=\phi_{3}\left(\mathbf{p}^{t-1}, \mathbf{m}^{t}, \boldsymbol{\eta}^{t-1}\right)$ $\geq \phi_{3}\left(\mathbf{p}^{t}, \mathbf{m}^{t}, \boldsymbol{\eta}^{t}\right)=\phi_{2}\left(\mathbf{p}^{t}, \mathbf{m}^{t}, \boldsymbol{\eta}^{t}\right)=\phi_{1}\left(\mathbf{p}^{t}, \mathbf{m}^{t}, \boldsymbol{\eta}^{t}\right)$. That is, $\phi_{1}\left(\mathbf{p}^{t-1}, \mathbf{m}^{t-1}, \boldsymbol{\eta}^{t-1}\right) \geq \phi_{1}\left(\mathbf{p}^{t}, \mathbf{m}^{t}, \boldsymbol{\eta}^{t}\right)$. It is obviously obtained that $\phi_{1}$ is monotonically non-increasing with respect to the iteration number and is lower bounded by a finite value. Therefore, Algorithm 3 is guaranteed to converge.

\section{Simulation results and discussion}

In this section, numerical simulations are performed by MATLAB software to validate the efficiencies and performance of the proposed algorithm, which is used to solve the joint task scheduling, offloading ratio and power control problem for latency-aware secure transmission in MEC-assisted HSR communication systems.

\subsection{Simulation configuration}

We consider a railway segment of length $L=500 \mathrm{~m}$ where $B=2 \mathrm{RBSs}$ are deployed [35]. A HSR with $K=2 \mathrm{VSs}$ is running from point $(100,0)$ with the speed of 100 $\mathrm{m} / \mathrm{s}$. Thus, the length of each time slot $T=2.5 \mathrm{~s}$ and the time slots $N=4$. The location of the eavesdropper is set to $(440,70)$. Figure 2 gives the corresponding coordinate positions of all BSs and VSs. The path loss between RBS and VS is modeled as $P L^{R B S}=141.1+36.4 \log 10\left(d_{k}[\mathrm{~km}]\right)$, where $d_{k}$ is the distance (in $\mathrm{km}$ ) between RBS and VS $k$. The noise power is $\sigma_{k}^{2}=\sigma_{e}^{2}=-110 \mathrm{dBm}$. The peak power budget of VS is set to $p_{k}^{\max }=30 \mathrm{dBm}$. It is assumed that the spectral bandwidth for offloading is $B=10 \mathrm{MHz}$, and the relevant Doppler interference factor $P_{\mathrm{ICI}}$ is $-19.4 \mathrm{~dB}$. The number of CPU cycles required per bit for local computing is $C_{k}[n]=10^{3}$ cycles/bit, the CPU cycle frequency $f_{k}[n]=3.4 \mathrm{GHz}$, and the effective capacitance coefficient

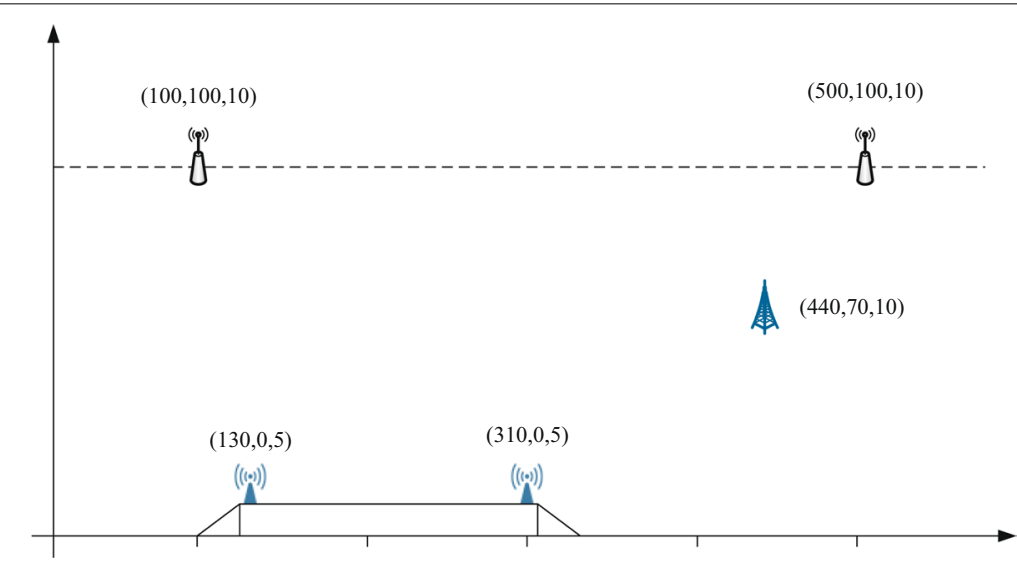

Fig. 2 Deployment locations of all RBSs and VSs 
Table 1 Simulation parameters

\begin{tabular}{ll}
\hline Parameters & Value \\
\hline Number of RBSs & 2 \\
Number of VSs & 2 \\
Speed of train & $100 \mathrm{~m} / \mathrm{s}$ \\
Number of time slots & 4 \\
Length of each time slot & $2.5 \mathrm{~s}$ \\
Maximum power of VS & $30 \mathrm{dBm}$ \\
Bandwidth & $10 \mathrm{MHz}$ \\
Doppler interference factor & $-19.4 \mathrm{~dB}$ \\
Number of CPU cycles per bit & $10^{3} \mathrm{cycles} / \mathrm{bit}$ \\
CPU cycle frequency & $3.4 \mathrm{GHz}$ \\
Effective capacitance coefficient & $10^{-28}$ \\
\hline
\end{tabular}

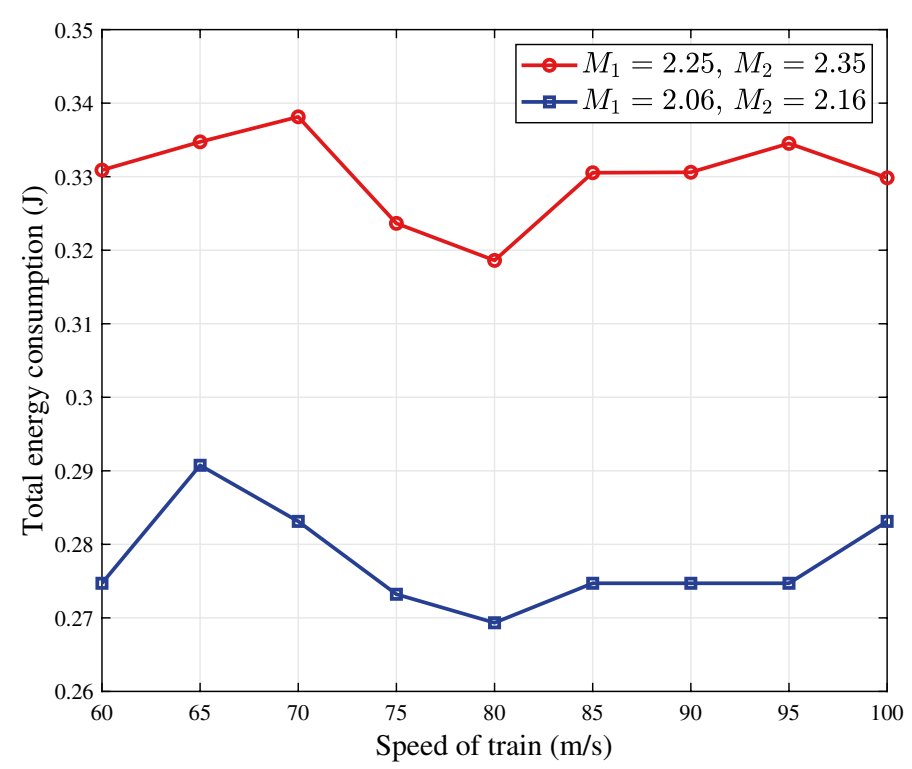

Fig. 3 Variation in total energy consumption versus the speed of train under different task sizes in the time $T$

is $\varpi_{k}[n]=10^{-28}$ [36]. Specifically, simulation parameters and values are listed in Table 1. Unless otherwise specified, these values are used in the sequel.

\subsection{Simulation results and analysis}

Figure 3 shows the variation in the total energy consumption versus the speed of train under different task sizes in the time $T$. The tasks in the above curve indicates are $M 1=2.25$ Mbits and $M 2=2.35$ Mbits, respectively, and the tasks in the following curve are $M 1=2.06 \mathrm{Mbits}$ and $M 2=2.16 \mathrm{Mbits}$, respectively. Figure 3 shows that under the same rate, the more the tasks to be processed, the more the total energy consumed by VSs. When the train speed is $80 \mathrm{~m} / \mathrm{s}$, the total energy consumption 
in both cases reaches the minimum values, which are $0.32 \mathrm{~J}$ and $0.27 \mathrm{~J}$, respectively. When the train speed is $70 \mathrm{~m} / \mathrm{s}$, the maximum energy consumption of the above curve is about $0.34 \mathrm{~J}$. When the train speed is $65 \mathrm{~m} / \mathrm{s}$, the maximum energy consumption of the following curve is about $0.29 \mathrm{~J}$. Because there are many factors involved in the energy consumption of VSs, the total energy consumption of VSs does not change monotonically with the rate.

Figure 4 gives the variation in the total energy consumption versus the speed of train under different distances between eavesdropper and VS, where the tasks are $M 1=2.25$ Mbits and $M 2=2.35$ Mbits, respectively. The short distance between eavesdropper and VS means that the coordinates of the eavesdropper are $[240,28,10]$, while the long distance between eavesdropper and VS means that the coordinates of the eavesdropper are $[440,70,10]$. As shown in Fig. 4, for the same rate, the energy consumption in the short distance is more than that in the long distance. This is because the secrecy rate in the short distance is less than that in the long distance. If the same offloading task is transmitted, more energy will be consumed. If fewer tasks are offloaded, the VS itself needs to process more local tasks and consume more energy, so it cannot obtain more task offloading gain. Therefore, offloading tasks need to be allocated according to the specific conditions.

Figure 5 illustrates the obtained task scheduling solution and the corresponding offloading ratio by performing the proposed algorithm; the eavesdropper is far away from VSs, where the tasks are $M 1=2.25 \mathrm{Mbits}$ and $M 2=2.35 \mathrm{Mbits}$, respectively. The left subfigure of Fig. 5 shows the amount of tasks to be processed by each VS in each time slot, and the right subfigure shows the task offloading ratio of each VS in each time slot. In the first time slot, each VS offloads $70 \%$ of the tasks to the RBS. In the second slot, VS1 and VS2 offload all tasks to RBS without performing any local computation. In the third time slot, VS1 offloads all tasks to RBS, and VS2 offloads $80 \%$ of tasks to RBS. In

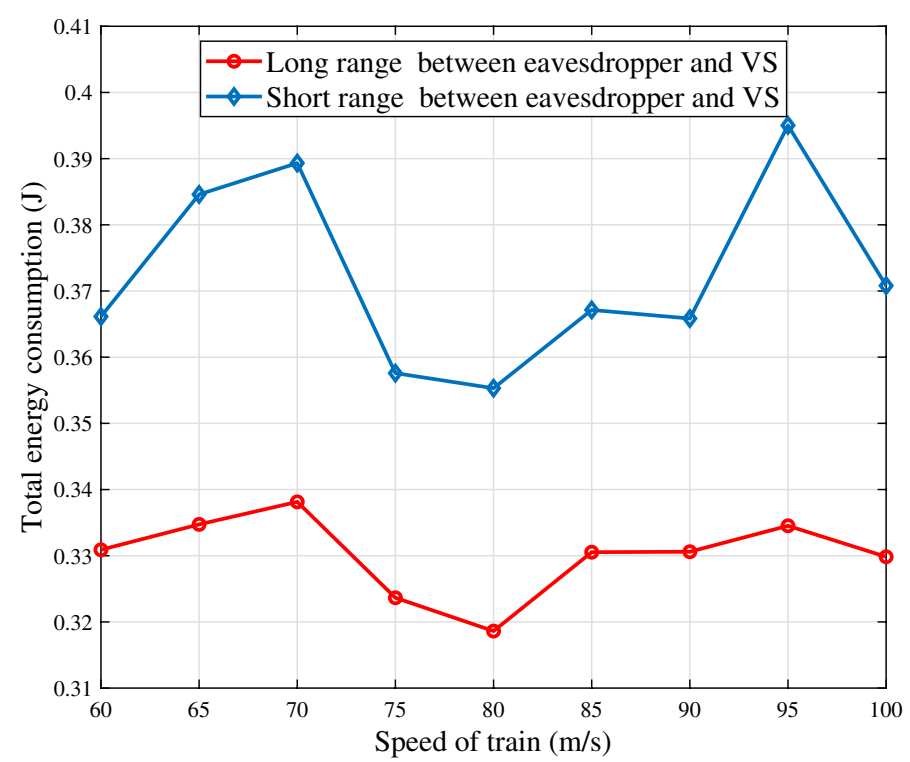

Fig. 4 Variation in total energy consumption versus the speed of train under different distances between eavesdropper and VS 

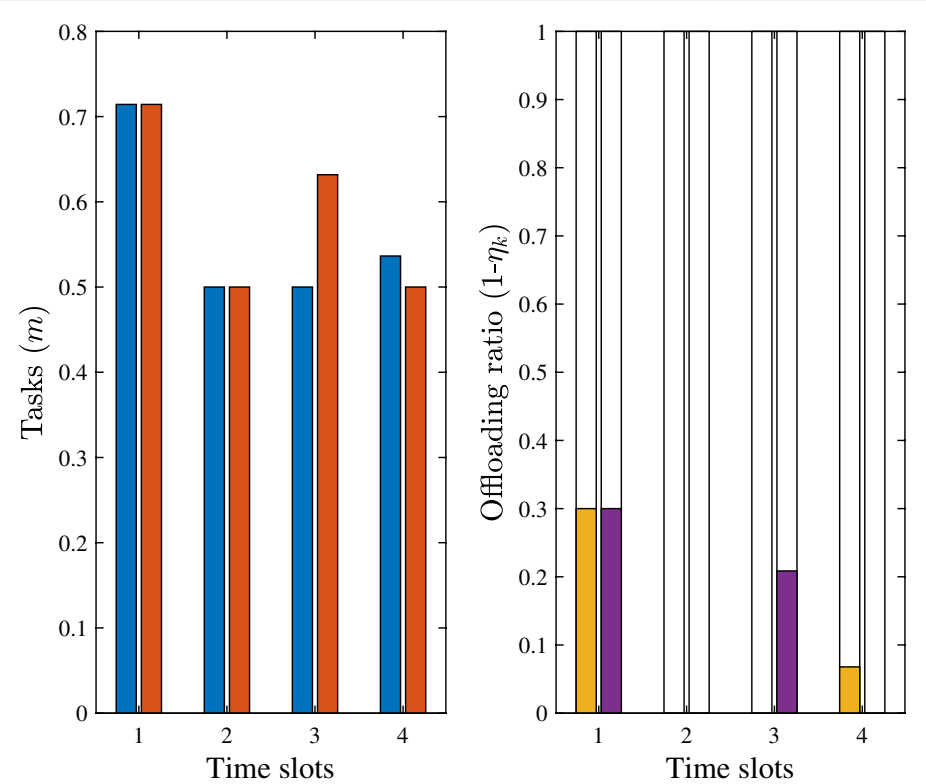

Fig. 5 Obtained task scheduling solution and the corresponding offloading ratio by performing the proposed algorithm
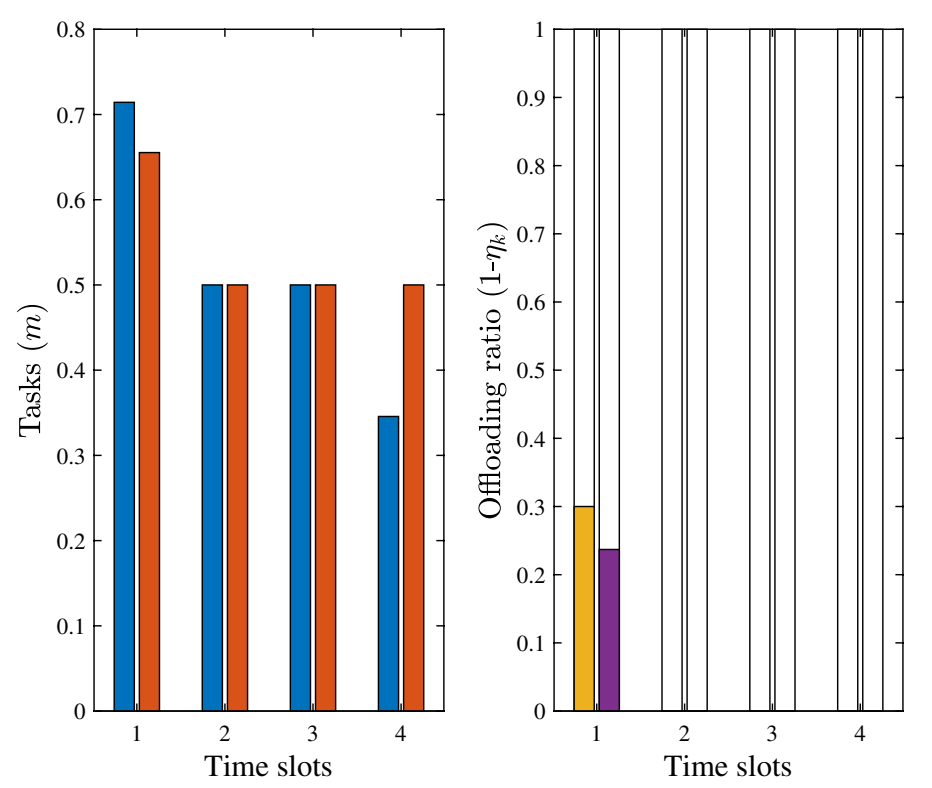

Fig. 6 Obtained task scheduling solution and the corresponding offloading ratio when the task size is set to a small value in the time $T$

the fourth time slot, VS1 offloads $95 \%$ of the tasks to RBS, and VS2 offloads all the tasks to RBS.

Figure 6 gives the obtained task scheduling solution and the corresponding offloading ratio by performing the proposed algorithm; the eavesdropper is far away from VSs, where the tasks are $M 1=2.06 \mathrm{Mbits}$ and $M 2=2.16 \mathrm{Mbits}$, respectively. As can be seen from the left subfigure of Fig. 6, there are the most tasks in the first time slot, and 
there are almost the same tasks in the other three time slots. In the first time slot, VS1 offloads $70 \%$ of the tasks to RBS and VS2 offloads $75 \%$ of the tasks to RBS. In the remaining three time slots, VS1 and VS2 offload all tasks to RBS without performing any local computation.

Compared with Figs. 5 and 7 shows the task scheduling solution and corresponding offloading rate obtained when the eavesdropper approaches the VSs, where the tasks are $M 1=2.25$ Mbits and $M 2=2.35$ Mbits, respectively. In the first time slot and the third time slot, the allocated tasks are the same as that in Fig. 5. In the second time slot, VS1 offloads $95 \%$ of the tasks to RBS, and VS2 offloads all the tasks to RBS. In the fourth time slot, VS1 and VS2 offload all tasks to RBS. As shown in Figs. 5, 6 and 7, in each time slot, when the allocated tasks by each VS are less than 0.5 Mbits, the VS offloads all tasks to the RBS. When the assigned tasks to each VS are greater than $0.5 \mathrm{Mbits}$, some tasks will be computed locally.

\section{Conclusion}

In this paper, MEC-assisted HSR wireless communication system was considered, and the energy consumption minimization of multiple VSs based on task offloading was studied. Due to the optimization variables including task scheduling, task offloading ratio and transmission power are coupled with each other; the optimization problem of minimizing energy consumption was intractable to solve. By fixing two of the three variables, we decomposed the original problem into three sub-problems. By introducing the auxiliary variables, the sub-problems were solved by interior point method and successive convex approximation algorithm. On this basis, an iterative energy consumption minimization algorithm was proposed.
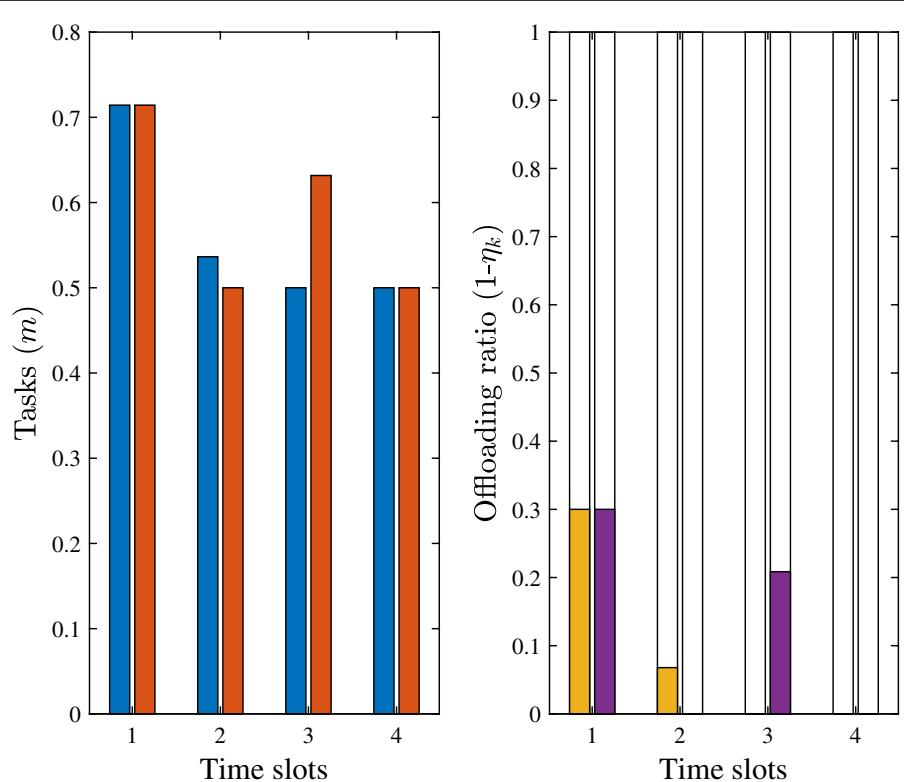

Fig. 7 Obtained task scheduling solution and the corresponding offloading ratio when the eavesdropper approaches VSs 


\section{Abbreviations}

IOT: Internet of thing; VR: Virtual reality; AR: Augmented reality; CPU: Central processing unit; MCC: Mobile cloud computing; MEC: Mobile edge computing; ETSI: European Telecommunications Standards Institute; HSR: High-speed railway; BPSO: Binary particle swarm optimization; PSO: Particle swarm optimization; MIMO: Multiple-in multiple-out; VS: Vehicular stations; RBS: Roadside base station; ICI: Inter-channel interference; QoS: Quality of service; SCA: Successive convex approximation..

\section{Authors' contributions}

The research and the outcome of this specific publication are result of a long cooperation between the authors about fundamentals and applications of secure HSR communication systems. For the present manuscript, we make a contribution to an iterative energy consumption minimization algorithm for MEC-assisted HSR wireless communication systems. All authors read and approved the final manuscript.

\section{Authors information}

Hao Xu received the B.S degrees in Communication Engineering from Lanzhou Jiaotong University, Lanzhou, China, in 2005, the M.S degrees in Communication and Information System from Lanzhou Jiaotong University in 2008 and in the PhD. Program of Traffic Information Engineering and Control in China Academy of Railway Sciences. He is currently an associate researcher who worked in Signal and Communication Research Institute of China Academy of Railway Sciences. He has been engaged in the research and development of high-speed railway communication system, comprehensive dispatching system, synthetic automation of marshalling yard and comprehensive scientific management during past 12 years.

Ke Li received the Ph.D. degree from Tianjin University, Tianjin, China, in 2011. He is currently an associate researcher at Signal and Communication Research Institute, China Academy of Railway Sciences Corporation Limited. His research interests include railway signal and communication, railway dynamic monitoring technology and intelligent data fusion technology.

Jianfeng Cheng received the B.S degrees in Industrial Automation from Hefei University of Technology, Hefei, China, in 1998, and the M.S degrees in Automation from University of Science and Technology of China, Hefei, China, in 2004. He is currently a researcher at Signal and Communication Research Institute, China Academy of Railway Sciences Corporation Limited. His research interests include railway signal and communication, railway dynamic monitoring technology and next-generation train control system.

Bo Jiang received B.S degrees in Communication Engineering from University of Science and Technology Beijing in 2011, the Master degree of Electrical Engineering from Stevens Institute of Technology in 2013 and currently in the PhD. Program of Transportation Planning and Management in China Academy of Railway Sciences. He is an assistant professor worked in Signal and Communication Research Institute of China Academy of Railway Sciences, focused on research and development of high-speed railway communication system, $5 \mathrm{G}$ dedicated networks for railway, and research of RSTT (Railway Radiocommunication Systems between Train and Trackside) in ITU during past 7 years.

Huai Yu received the B.S degrees in Electronic Information Science and Technology from Guizhou University, Guiyang, China, in 2010, and the M.S degrees in Traffic Information Engineering and Control from China Academy of Railway Sciences, Beijing, China, in 2014. He is currently an assistant research fellow who worked in Signal and Communication Research Institute of China Academy of Railway Sciences. He has been engaged in the research and development of railway signal and communication system, railway transportation and dispatching, and system quality control during past years.

\section{Funding}

This research was supported in part by the key Programs of Technological R\&D of China Academy of Railway Sciences under the Grant 1951TH6804.

\section{Declarations}

\section{Consent for publication}

Informed consent was obtained from all authors included in the study.

\section{Competing interests}

The authors declare that they have no competing interests.

Received: 14 October 2021 Accepted: 14 November 2021

Published online: 27 November 2021

\section{References}

1. Y. Siriwardhana, P. Porambage, M. Liyanage, M. Ylianttila, A survey on mobile augmented reality with $5 G$ mobile edge computing: architectures, applications, and technical aspects. IEEE Commun. Surv. Tutor. 23(2), 1160-1192 (2021)

2. Y. Liu, M. Peng, G. Shou, Y. Chen, S. Chen, Toward edge intelligence: multiaccess edge computing for $5 \mathrm{G}$ and internet of things. IEEE Int. Things J. 7(8), 6722-6747 (2020)

3. X. Liu, X. Zhang, Rate and energy efficiency improvements for 5G-based loT with simultaneous transfer. IEEE Int. Things J. 6(4), 5971-5980 (2019)

4. M. Feng, M. Krunz, W. Zhang, Joint task partitioning and user association for latency minimization in mobile edge computing networks. IEEE Trans. Veh. Technol. 70(8), 8108-8121 (2021) 
5. X. Liu, X. Zhang, M. Jia, 5G-based green broadband communication system design with simultaneous wireless information and power transfer. Phys. Commun. 28, 130-137 (2018)

6. X. Liu, X. Zhang, NOMA-based resource allocation for cluster-based cognitive industrial internet of things. IEEE Trans. Ind. Inform. 16(8), 5379-5388 (2020)

7. A.U.R. Khan, M. Othman, S.A. Madani, S.U. Khan, A survey of mobile cloud computing application models. IEEE Commun. Surv. Tutor. 16(1), 393-413 (2014)

8. A.U.R. Khan, M. Othman, F. Xia, A.N. Khan, Context-aware mobile cloud computing and its challenges. IEEE Cloud Comput. 2(3), 42-49 (2015)

9. P. Si, Q. Zhang, F.R. Yu, Y. Zhang, QoS-aware dynamic resource management in heterogeneous mobile cloud computing networks. China Commun. 11(5), 144-159 (2014)

10. K. Elgazzar, P. Martin, H.S. Hassanein, Cloud-assisted computation offloading to support mobile services. IEEE Trans. Cloud Comput. 4(3), 279-292 (2016)

11. G.H.S. Carvalho, I. Woungang, A. Anpalagan, M. Jaseemuddin, Analysis of joint parallelism in wireless and cloud domains on mobile edge computing over 5G systems. J. Commun. Netw. 20(6), 565-577 (2018)

12. Z. Sanaei, S. Abolfazli, A. Gani, R. Buyya, Heterogeneity in mobile cloud computing: taxonomy and open challenges. IEEE Commun. Surv. Tutor. 16(1), 369-392 (2014)

13. X. Liu, X. Zhai, W. Lu, C. Wu, QoS-guarantee resource allocation for multibeam satellite industrial internet of things with NOMA. IEEE Trans. Ind. Inform. 17(3), 2052-2061 (2021)

14. Y. Mao, C. You, J. Zhang, K. Huang, K.B. Letaief, A survey on mobile edge computing: the communication perspective. IEEE Commun. Surv. Tutor. 19(4), 2322-2358 (2017)

15. X. Ding, J. Wu, Study on energy consumption optimization scheduling for internet of things. IEEE Access $\mathbf{7}$, 70574-70583 (2019)

16. P. Mach, Z. Becvar, Mobile edge computing: a survey on architecture and computation offloading. IEEE Commun. Surv. Tutor. 19(3), 1628-1656 (2017)

17. L. Cui, Joint optimization of energy consumption and latency in mobile edge computing for internet of things. IEEE Int. Things J. 6(3), 4791-4803 (2019)

18. C. Meng, G. Wang, B. Yan, Y. Li, Energy efficiency optimization for secure SWIPT system. IEICE Trans. Commun. 103(5), E582-B-590 (2020)

19. C. Meng, G. Wang, X. Dai, Secure energy-efficient transmission for SWIPT intelligent connected vehicles with imperfect CSI. IEEE Access 7, 154649-154658 (2019)

20. M.M. Mowla, I. Ahmad, D. Habibi, Q.V. Phung, A green communication model for $5 \mathrm{G}$ systems. IEEE Trans. Green Commun. Netw. 1(3), 264-280 (2017)

21. W. Labidi, M. Sarkiss, M. Kamoun, in Proceedings of the International Conference on Telecommunication (ICT), EnergyOptimal Resource Scheduling and Computation Offloading in Small Cell Networks (2015), pp. 313-318

22. M. Deng, H. Tian, B. Fan, in Proceedings of the IEEE International Conference on Communication Workshops (ICC), FineGranularity Based Application Offloading Policy in Cloud-enhanced Small Cell Networks (2016), pp. 638-643

23. C. You, K. Huang, H. Chae, Energy efficient mobile cloud computing powered by wireless energy transfer. IEEE J. Sel. Areas Commun. 34(5), 1757-1771 (2016)

24. S.E. Mahmoodi, R.N. Uma, K.P. Subbalakshmi, Optimal joint scheduling and cloud offloading for mobile applications. IEEE Trans. Cloud Comput. 7(2), 301-313 (2019)

25. W. Zhang, Y.Wen, D.O. Wu, Collaborative task execution in mobile cloud computing under a stochastic wireless channel. IEEE Trans. Wirel. Commun. 14(1), 81-93 (2015)

26. W. Labidi, M. Sarkiss, M. Kamoun, in Proceedings of the IEEE International Conference on Wireless Mobile Computer Network Communication (WiMob), Joint Multi-user Resource Scheduling and Computation Offloading in Small Cell Networks (2015) pp. 794-801

27. K. Zhang, Energy-efficient offloading for mobile edge computing in $5 \mathrm{G}$ heterogeneous networks. IEEE Access 4 5896-5907 (2016)

28. S. Li, H. Ge, X. Chen, L. Liu, H. Gong, R. Tang, in Proceedings of the 2021 IEEE 6th International Conference on Cloud Computing and Big Data Analytics (ICCCBDA), Computation Offloading Strategy for Improved Particle Swarm Optimization in Mobile Edge Computing (2021), pp. 375-381

29. C. You, K. Huang, in Proceedings of the IEEE Global Communication Conferecne (GLOBECOM), Multiuser Resource Allocation for Mobile Edge Computation Offloading (2016), pp. 1-6

30. K. Wang, K. Yang, C.S. Magurawalage, Joint energy minimization and resource allocation in C-RAN with mobile cloud. IEEE Trans. Cloud Comput. 6(3), 760-770 (2018)

31. S. Sardellitti, G. Scutari, S. Barbarossa, Joint optimization of radio and computational resources for multicell mobileedge computing. IEEE Trans. Signal Inf. Process. Over Netw. 1(2), 89-103 (2015)

32. X. Lyu, H. Tian, C. Sengul, P. Zhang, Multiuser joint task offloading and resource optimization in proximate clouds. IEEE Trans. Veh. Technol. 66(4), 3435-3447 (2017)

33. W. Zhang, Energy-optimal mobile cloud computing under stochastic wireless channel. IEEE Trans. Wirel. Commun 12(9), 4569-4581 (2013)

34. B. Ji, Y. Li, D. Cao, C. Li, S. Mumtaz, D. Wang, Secrecy performance analysis of UAV assisted relay transmission for cognitive network with energy harvesting. IEEE Trans. Veh. Technol. 69(7), 7404-7415 (2020)

35. Q. Zhang, H. Zheng, Z. Zhong, in Proceedings of the IEEE ehicular Technology Conference (VTC2020-Fall), Energy-Aware Dynamic Computation Offloading in High-Speed Railway Networks with D-TDD (2020), pp. 1-6

36. J. Wang, H. Yang, M. Cheng, J. Wang, M. Lin, J. Wang, Joint optimization of offloading and resources allocation in secure mobile edge computing systems. IEEE Trans. Veh. Technol. 69(8), 8843-8854 (2020)

\section{Publisher's Note}

Springer Nature remains neutral with regard to jurisdictional claims in published maps and institutional affiliations. 